\title{
About the Existence \\ of Solutions of a Boundary Value Problem for a Carathéodory Differential System
}

\author{
G. Anichini and G. Conti
}

\begin{abstract}
A boundary value problem of the form $x^{\prime}=f(t, x), x \in S$ is considered where $f$ is a Carathéodory function and $S$ is a suitable boundary constraint. A related integral equation with an exact number of solutions is studied, in order to show the existence of solutions of the boundary value problem through a fixed point theorem for $w$-maps in the Darbo sense.
\end{abstract}

Keywords: Solutions set, isolated points, number of solutions, integral equations, boundary value problems

AMS subject classification: 34 B 15, 45 G 10, 46 H 30

\section{Introduction}

In many papers the question of determining the exact number of solutions of a differential problem is considered (see, for istance, $[1,10,13]$ and the references therein). In this paper such an argument is used in order to get an existence result for a general boundary value problem of the type

$$
\left.\begin{array}{l}
x^{\prime}=f(t, x) \quad \text { for a.e. } t \in I=[a, b] \subset \mathbb{R}, x \in \mathbb{R}^{n} \\
x \in S \quad\left(S \subset A C\left(I, \mathbb{R}^{n}\right)\right) .
\end{array}\right\}
$$

To that purpose the boundary value problem (BV), where $f$ is a Carathéodory function, will, in some sense, be connected to an integral equation which is known to have an exact number of solutions.

The technique we are going to employ consists essentially in the use of the solution set of some (hopefully easier) related differential or integral equation in order to obtain the assumptions needed to apply a suitable fixed point theorem. Such an approach worked as well in $[4,5]$.

G. Anichini: Univ. Modena, Dept. Math., Via Campi 213/b, 41100 - Modena, Italy

G. Conti: Inst. Math., Fac. Archit., Via dell'Agnolo 14, 50122 - Firenze, Italy

Work done under the auspices of $40 \%$ MURST-Research Project "Equazioni Differenziali Ordinarie e Applicazioni" 


\section{Preliminaries}

Let $X$ and $Y$ be metric spaces. $B_{r}\left(x_{0}\right)$ will denote an $r$-ball (in the space $X$ ), i.e. the set $\left\{x \in X: d\left(x, x_{0}\right)<r\right\}$ where $x_{0}$ is any point in $X$ and $d$ is a metric on $X$. If $A$ and $B$ are non-empty closed subsets of $X$ and we define $d_{X}(b, A)=\inf _{a \in A} d_{X}(b, a)$, where $d_{X}$ is a semi-distance defining a topology on $X$, then it is possible to define a semi-distance between $A$ and $B$ by $\delta_{X}(A, B)=\sup _{b \in B} d_{X}(b, A)$. If $A$ and $B$ are compact subsets of the space $X$, we define the Hausdorff metric $D_{X}$ in $X$ by putting $D_{X}(A, B)=\max \left(\delta_{X}(A, B), \delta_{X}(B, A)\right)$.

Definition 1. A multi-valued mapping $G: X \rightarrow Y$ is said to be upper semicontinuous at the point $x_{0} \in X$ if, for any open set $V \supset G\left(x_{0}\right)$, there exists a neighborhood $U$ of $x_{0}$ such that $G(x) \subset V$ for any $x \in U$.

If, for every $x \in X, G$ is upper semicontinuous at $x$ and $G(x)$ is a compact set, then $G$ is said to be upper semicontinuous on $X$. If an upper semicontinuous mapping $G$ sends bounded sets into relatively compact sets, then it is said to be compact. Actually, an operator $G: X \rightarrow Y$ is a compact one if $G(B)$ lies in a compact set of $Y$ for any bounded subset $B \subset X$. So if the set-valued function $G$ has compact values, we can say that $G$ is upper semicontinuous at the point $x_{0}$ if and only if for every $\varepsilon>0$ there is a neighborhood $U$ of $x_{0}$ such that, for every $x \in U, \delta_{X}\left(G(x), G\left(x_{0}\right)\right)<\varepsilon$.

We want also to recall that $G: X \longrightarrow Y$ is called a closed graph operator if from $x_{n} \rightarrow x_{0}$ in $X$ and $y_{n} \rightarrow y_{0}$ in $Y$ with $y_{n} \in G\left(x_{n}\right)$ it follows $y_{0} \in G\left(x_{0}\right)$. If $G(x)$ is a closed set for all $x \in X$ and $G(X)$ is a relatively compact set, then $G$ is upper semicontinuous if and only if $G$ is a closed graph operator.

Definition 2 (see [15]). A function $(t, x, y) \rightarrow f(t, x, y)$ is said to be a Carathéodory function (and we shall write $f \in \operatorname{Car}\left(I \times \mathbb{R}^{n} \times \mathbb{R}^{n}, \mathbb{R}^{n}\right)$ where $I$ is a real interval), if the following properties hold:

a) $t \rightarrow f(t, x, y)$ is a measurable function for all $(x, y) \in \mathbb{R}^{n} \times \mathbb{R}^{n}$.

b) $(x, y) \rightarrow f(t, x, y)$ is a continuous function for a.a. $t \in I$.

c) For every $M>0$ there is a function $h_{M} \in L^{1}\left(I, \mathbb{R}^{+}\right)$such that, for a.a. $t \in I$ and for every $(x, y)$ with $|(x, y)| \leq M$, we have $|f(t, x, y)| \leq h_{M}(t)$, where the $R^{n}$ vector norm $\|\cdot\|$ is simply denoted by $|\cdot|$.

Definition 3 (see $[8,11,12,20]$ ). A multi-valued upper semicontinuous map $G: X \longrightarrow Y$ is called a weighted carrier (for short: a $w$-carrier in the Darbo sense, with weights in a commutative ring $K)$, if to each $x$ and $y \in G(x)$ and any open subset $V \subset Y$ with $\partial V \cap G(x)=\emptyset$ an integer number $m_{V}(y, G(x))$ belonging to $K$ and called multiplicity or weight is assigned in such a way that the following properties hold:

(i) $m_{V}(y, G(x))$ is a locally constant function of $x$.

(ii) $m_{V}(y, G(x))=0$ if $\partial V \cap G(x)=\emptyset$.

(iii) $m_{V_{1} \cup V_{2}}(y, G(x))=m_{V_{1}}(y, G(x))+m_{V_{2}}(y, G(x))-m_{V_{1} \cap V_{2}}(y, G(x))$ for any open subsets $V_{1}, V_{2} \subset Y$. 
When the $w$-carrier maps any point of $X$ into a finite number of points of the range space $Y$, it is called a weighted map (for short: a w-map in the Darbo sense [11]). In this case the property (i) above can be stated as

$$
\sum_{y \in G(x) \cap V} m_{V}(y, G(x))=\sum_{y^{\prime} \in G\left(x^{\prime}\right) \cap V} m_{V}\left(y^{\prime}, G\left(x^{\prime}\right)\right)
$$

whenever $x^{\prime}$ is close enough to $x$.

Remark. If, given an upper semicontinuous map $G: X \longrightarrow Y$, the set $G(x)$ is connected for any $x \in X$, then $G$ becames a $w$-carrier by assigning multiplicity $1 \in K$ to $F(x)$. In particular, any continuous single-valued map is a $w$-carrier.

The number

$$
i(G(x), V)=\sum_{y \in G(x) \cap V} m_{V}(y, G(x))
$$

is called the index of the set $G(x)$ in $V$. When $V$ is a connected set, the number $i(G(x), V))$ does not depend on $x \in X$. In this case that number will be called the index of $G$ and it will be denoted by $i(G)$. Other properties and results concerning $w$-maps are widely considered in $[8,11,12,20]$. In particular, in [8: Section 3] and in [11] there is also a number of examples of $w$-carriers.

A set $X$ which is the homeomorphic image of a geometric polyhedron (i.e. the union of a finite number of geometric simplexes) will be shortly called a polyhedron. We say that $X$ is an acyclic set if it is acyclic in positive dimension, in the sense of the Čech homology with coefficients in a ring $K$ (i.e. the $n$-homology group $H^{n}(X)$ is trivial for $n>0)$. We refer to [6] for a detailed introduction of the needed algebraic topological tools.

Finally, the following fixed point result will be crucial in the sequel.

Proposition 1 (see [20]). Let $X$ be any acyclic polyhedron over a ring $K$. Then any $w$-carrier $G: X \longrightarrow X$ for which $i(G) \neq 0$ has a fixed point.

Remark. When the domain of the function $G$ is an acyclic polyhedron, then the index $i(G)$ introduced above is essentially the Lefschetz number of the function $G$, widely considered in the literature (see, for istance, $[8,12,20]$ and the references therein).

Definition 4 (see [19: Chapter 3]). An integral equation of the form

$$
P x(t)=\int_{I} k(t, s, x(s)) d s
$$

where $I \subset \mathbb{R}$ is an interval and $x: \mathbb{R} \rightarrow \mathbb{R}$ is a measurable function, is the simplest and most important nonlinear integral equation. It is usually called Urysohn integral equation. tion

A particular case of an Urysohn integral equation is the Hammerstein integral equa-

$$
A x(t)=\int_{I} k(t, s) f(s, x(s)) d s,
$$


where the function $(t, x) \rightarrow f(t, x)$ defined for $t \in I$ is continuous with respect to $x$ for a.a. $t \in I$ and measurable with respect to $t$ for a.a. $x$, and the kernel $(t, s) \rightarrow k(t, s)$ is measurable with respect to $(t, s)$.

In the next result which will be very useful in the proof of our main Theorem 1 other properties of an Hammerstein integral equation will be introduced.

Proposition 2 (see [21: Chapter 7]). The boundary value problem

$$
\left.\begin{array}{l}
x^{\prime}=g(t, x, q) \quad \text { a.e } \quad t \in I=[a, b] \subset \mathbb{R} \\
x \in S_{1},
\end{array}\right\}
$$

where $g \in \operatorname{Car}\left(I \times \mathbb{R}^{n} \times \mathbb{R}^{n}, \mathbb{R}^{n}\right)$ and $S_{1} \subset A C\left(I, \mathbb{R}^{n}\right)$ is a suitable bounded and closed subset, is equivalent to an integral equation like

$$
x=A_{q}(x) \equiv A(x, q)
$$

for all $q \in Q \subset A C\left(I, \mathbb{R}^{n}\right)$, where $(x, q) \rightarrow A(x, q)$ is a compact operator for all $q \in Q$.

As a matter of fact we know (see [21: p. 159]) that a boundary value problem (with either homógeneous or non-homogeneous boundary conditions) could be written in operator form as $L x=g$ where $L$ is a linear ordinary differential operator whose domain is the set of all absolutely continuous (with respect to $x$ ) and measurable (with respect to $t$ ) functions which are in $S_{1}$. Then, in conformity with the above discussion, it is possible to see that the differential operator (together with its domain or, equivalently, together with the associated boundary conditions) has an inverse in the form of an integral operator. So we can consider the Hammerstein integral equation.

$$
x(t)-\int_{I} k(t, s) g(s, x(s) ; q(s)) d s=0
$$

where $(t, s) \longrightarrow k(t, s)$ is the so-called Green function of the differential operator. (The Green function of a differential operator is an everywhere continuous function whose derivative has a jump discontinuity for $t=s$ and it is the kernel of an integral operator which inverts the differential operator $L$.) So, if we write the previous Hammerstein integral equation as

$$
A_{q} x(t)=\int_{I} k(t, s) g(s, x(s), q(s)) d s
$$

or, shortly,

$$
A_{q} x=\bar{k} \overline{g_{q}}(x)
$$

where $\bar{k} u(t)=\int_{I} k(t, s) u(s) d s$ is a linear integral operator and $\overline{g_{q}}(t)=g(t, x(t), q(t))$ a nonlinear superposition operator, then the equivalence between the given boundary value problem $(\mathrm{BV})_{q}$ and the integral equation (IE) can be positively established.

The integral operator $A_{q}$ will be supposed to be compact - as we shall see in the application this is not a strong assumption. As a matter of fact, if the function $x$ belongs to a Lebesgue space $L_{q}(I)$ and $p$ is a real number such that $\frac{1}{p}+\frac{1}{q}=1$, then by writing the Hammerstein equation as $x=\bar{k} \overline{g_{q}}(x)$ with $x \in L_{p}(I)$, it is known that, for $p>1$, $\bar{k}$ is a linear compact operator (see [22: Chapter 2] or [19: Chapter 1]), because of the continuity of the Green function. Now suitable conditions on the function $g$ (e.g., growth conditions) allows to say that also $A_{q}=\bar{k} \overline{g_{q}}$ is a compact operator.

Finally, in the next definition we want to recall the concept of topological degree. 
Definition 5 (see [13: Chapter 1] or [22: p. 291]). Let $\Omega \subset \mathbb{R}^{n}$ be a bounded and open set and $f: \bar{\Omega} \rightarrow \mathbb{R}^{n}$ a continuous function. Then if $y \in \mathbb{R}^{n} \backslash f(\partial \Omega)$, there is only one function

$$
\operatorname{deg}:\{(f, \Omega, y)\} \longrightarrow \mathbb{Z}
$$

with the following properties:

1) $\operatorname{deg}(i d, \Omega, y)=1$, where $i d(y)=y$ for all $y \in \Omega$.

2) $\operatorname{deg}(f, \Omega, y)=\operatorname{deg}\left(f, \Omega_{1}, y\right)+\operatorname{deg}\left(f, \Omega_{2}, y\right)$ whenever $\Omega_{1}, \Omega_{2} \subset \Omega$ are disjoint open subsets such that $y \notin f\left(\bar{\Omega} \backslash \Omega_{1} \cup \Omega_{2}\right)$.

3) $\operatorname{deg}(h(t, \cdot), \Omega, y(t))$ is independent of $t \in[0,1]$ whenever $h:[0,1] \times \bar{\Omega} \rightarrow \mathbb{R}^{n}$ and $y:[0,1] \rightarrow \mathbb{R}^{n}$ are continuous and $y(t) \notin h(t, \partial \Omega)$ for all $t \in[0,1]$.

The number $\operatorname{deg}(f, \Omega, \cdot)$ is called the topological degree of $f$ with respect to $\Omega$.

\section{Results}

In the statement of the main theorem we want to consider the boundary value problems $(\mathrm{BV})$ and $(\mathrm{BV})_{q}$ under the following assuptions:

i) $f \in \operatorname{Car}\left(I \times \mathbb{R}^{n}, \mathbb{R}^{n}\right)$ and $g \in \operatorname{Car}\left(I \times \mathbb{R}^{n} \times \mathbb{R}^{n}, \mathbb{R}^{n}\right)$ are such that $f(t, c)=g(t, c, c)$ for almost all $c \in \mathbb{R}^{n}$.

ii) $Q \subset A C\left(I, R^{n}\right)$ is a bounded, closed and convex set and $S_{1} \subset Q \cap S$ is a closed set.

Let us recall that in Proposition 2 the equivalence between a boundary value problem and an integral equation was introduced.

Theorem 1. Let us assume that the sets $Q$ and $S_{1}$ of ii) are given in such a way that the boundary value problem $(\mathrm{BV})_{q}$ is equivalent to some integral equation of the form (IE) where $(x, q) \rightarrow A(x, q): \Omega \times Q \rightarrow S_{1} \subset A C\left(I, R^{n}\right), A(x, q)$ is a compact operator for each $q \in Q$ and it is such that $\operatorname{deg}\left(I-A_{q}, \Omega, 0\right) \neq 0$ for some (and hence for all) $q \in Q$ and some open and convex subset $\Omega \subset A C\left(I, \mathbb{R}^{n}\right)$. Further, let $\Sigma: Q \rightarrow 2^{Q}$ be the multi-valued operator which maps each $q \in Q$ into the set of solutions of the integral equation (IE).

Then if the set $\Sigma(q)$ is discrete for each $q \in Q$, the boundary value problem (BV) has at least one solution.

Proof. Let $\Sigma: Q \rightarrow 2^{S_{1}}$ be the given multi-valued operator. In order to apply the Darbo fixed point theorem (see [11: p. 393]), we need to show that $\Sigma$ is

- upper semicontinuous

- compact

- a $w$-map in the Darbo sense.

To the first purpose let us consider a sequence $q_{n} \in Q_{1}=\overline{c o} S_{1}$ (i.e. the convex closure of the bounded set $\left.S_{1}\right)$ such that $\lim _{n} q_{n}=q_{0}$, and a sequence $x_{n} \in \Sigma\left(Q_{1}\right)$ such that $\lim _{n} x_{n}=x_{0}$. Now it will be enough to show that $x_{0} \in \Sigma\left(q_{0}\right)$ in order to apply the 
closed graph property and to get both the upper semicontinuity of the operator $\Sigma$ and the compactness of the set $\Sigma\left(q_{0}\right)$.

Let us consider the sequence of Hammerstein integral equations

$$
x_{n}=\int_{I} k(t, s) g\left(s, x_{n}(s), q_{n}(s)\right) d s
$$

We observe that, by the continuity with respect to the second and third variable of the function $g$, it is possible to apply the Lebesgue dominated convergence theorem to get

$$
\begin{aligned}
x_{0}(t) & =\lim _{n} x_{n} \\
& =\lim _{n} \int_{I} k(t, s) g\left(s, x_{n}(s), q_{n}(s)\right) d s \\
& =\int_{I} \lim _{n} k(t, s) g\left(s, x_{n}(s), q_{n}(s)\right) d s \\
& =\int_{I} k(t, s) g\left(s, x_{0}(s), q_{0}(s)\right) d s
\end{aligned}
$$

by using also the continuity of the kernel operator $(t, s) \rightarrow k(t, s)$. The latter equality means that $t \rightarrow x_{0}(t)$ is a solution of the Hammerstein equation $x=A_{q_{0}}(x)$ or, equivalently, that $x_{0} \in \Sigma\left(q_{0}\right)$.

For the second purpose let us firstly observe that a function $t \rightarrow \bar{x}(t)$ belongs to the set $\Sigma(\bar{q})$ for some $\bar{q} \in Q$ if and only if the equality $\bar{x}=A_{\bar{q}}(\bar{x})$ holds. Then, in order to get the compactness of the operator $\Sigma$ we need to show that, for any bounded subset $B \subset Q, \Sigma(B)$ is a subset of some compact set of $2^{Q}$. By hypothesis we know that $A_{q}$ is a compact operator for each $q \in Q$. On the other hand, this statement is equivalent to say that $A_{q}(B)$ lies in a compact subset of $2^{Q}$ for any bounded subset $B \subset Q$. So it is enough to take the bounded set $Q_{1}=\overline{c o}\left(S_{1}\right)$ in order to get the compactness of the operator $A_{q}$.

Now it is enough to show that $x_{0} \in \Sigma\left(q_{0}\right)$ inasmuch as the closed graph property allows us to say that $\Sigma$ is an upper semicontinuous operator and that $\Sigma\left(q^{\circ}\right)$ is a compact set. We have only to prove that $\Sigma$ is a $w$-map. Let $q \in Q$ be fixed and take any $x \in \Sigma(q)$. By hypothesis we know that, for each $q \in Q$, the integral operator $A_{q}$ is compact: since $x$ is an isolated solution of the integral equation it will be possible to have an open neighborhood $\Omega_{1} \subset A C\left(I, \mathbb{R}^{n}\right)$ such that $\Omega_{1} \cap \Sigma(q)=\{x\}$. Let us define $m_{\Omega_{1}}(x, \Sigma(q))=\operatorname{deg}\left(I-A_{q}, \Omega_{1}, 0\right)$. To show that this integer number is the weight $m_{\Omega_{1}}(x, \Sigma(q))$ we need to say that $\Sigma$ shares the properties of a $w$-map. We can say that this number does not depend on the choice of the set $\Omega_{1}$ because of the third property of the topological degree. Let now $W$ be an open subset of $A C\left(I, \mathbb{R}^{n}\right)$ such that $\Sigma(q) \cap \partial W \neq \emptyset$. The upper semicontinuity of $\Sigma$ implies the existence of some ball $B_{r}(q)$ such that $\Sigma\left(q^{\prime}\right) \cap \partial W \neq \emptyset$ for all $q^{\prime} \in B_{r}(q)$. Then a homotopy $h: W \times[0,1] \rightarrow A C\left(I, \mathbb{R}^{n}\right)$ can be defined as $h(x, t)=A\left(x, t q+(1-t) q^{\prime}\right)$ for $q^{\prime} \in B_{r}(q)$. We have $t q+(1-t) q^{\prime} \in B_{r}(q)$ for all $t \in[0,1]$. Thus the given homotopy is an admissible 
one between $A_{q} \backslash W$ and $A_{q}^{\prime} \backslash W$. The latter result and the second property of the topological degree imply

$$
\sum_{x \in \Sigma(q) \cap W} m_{\Omega_{1}}(x, \Sigma(q))=\operatorname{deg}\left(A_{q}, W, 0\right)=\operatorname{deg}\left(A_{q}^{\prime}, W, 0\right)=\sum_{x \in \Sigma\left(q^{\prime}\right) \cap W} m_{\Omega_{1}}\left(y, \Sigma\left(q^{\prime}\right)\right) .
$$

So $\Sigma$ has the properties of a $w$-map where $i(\Sigma)=\operatorname{deg}\left(I-A_{q}, \Omega, 0\right)$. This allows us to use the fixed point theorem quoted in Proposition 1 and to get the required result

\section{Applications}

Let us consider the two-point boundary value problem

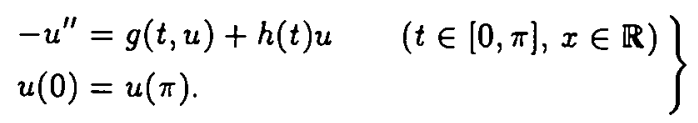

The following assumptions on the function $g:[0, \pi] \times \mathbb{R} \rightarrow \mathbb{R}$ are here considered:

i) $(t, x) \rightarrow g(t, x)$ is a Carathéodory function (i.e. measurable with respect to $t$ for each $u$ and continuous with respect to $u$ for a.a. $t \in(0, \pi))$ such that

$$
\lim _{x \rightarrow+\infty} \frac{g(t, x)}{x}=g(+) \quad \text { and } \quad \lim _{x \rightarrow-\infty} \frac{g(t, x)}{x}=g(-)
$$

for a.a. $t \in(0, \pi)$ and the closed interval $(g(+), g(-))$ or $((g(-), g(+))$ contains the first (simple) eigenvalue of the problem

$$
\left.\begin{array}{l}
-v^{\prime \prime}=\lambda v \\
v(0)=v(\pi)
\end{array}\right\}
$$

and $v(t) \geq 0$ for all $t \in(0, \pi)$.

ii) $\sup _{x \in \mathbb{R}}\left|\frac{g(\cdot, x)}{x}\right|$ is an (essentially) bounded function.

iii) $u \rightarrow g(\cdot, u)$ is a strictly convex and increasing function.

Let $Q$ denote the subset of $L^{2}(0, \pi)$ defined as

$$
Q=\left\{u \in L^{2}(0, \pi):\|u\|+\left\|u^{\prime}\right\| \leq \bar{M} \text { and } u(0)=u(\pi)=0 .\right\}
$$

It is easily seen that $Q$ is a closed and convex set. Afterwards, if $\phi$ is an eigenvalue of the second order differential problem considered in i), i.e. $A \phi=\lambda \phi$ and $\|\phi\|=1$, let us denote by $\phi^{\perp}$ the orthogonal complement of $\phi$ in $L^{2}(0, \pi)$. The above assumptions on the function $g$ allow us to state the following result. 
Theorem 2 (see (14: Section 1.2]). Let us assume that the conditions i) - iii) are true. Then, for a.a. $t \in(0, \pi)$, for each $h_{0} \in \phi^{\perp}$ and for each function $h \in L^{2}(0, \pi)$ such that $h q=h_{0}+r \phi \quad(r \in \mathbb{R}, q \in Q)$ there exists $a \in \mathbb{R}$ such that the differential problem

$$
\left.\begin{array}{l}
-u^{\prime \prime}=g(t, u)+h(t) q(t) \\
u(0)=u(\pi)
\end{array}\right\}
$$

has exactly one solution if $r=a$ and exactly two solutions if $r<a$.

Now we want to study the properties of the operator

$$
\sum:\left(q, q^{\prime}, q^{\prime \prime}\right) \longrightarrow \sum\left(q, q^{\prime}, q^{\prime \prime}\right)
$$

which associates to every function in $Q \subset L^{2}(0, \pi)$ the solution set of the problem $(\mathrm{P})_{q}$. Let $(t, s) \rightarrow G(t, s)$ denote the Green function of the homogeneous problem

$$
\left.\begin{array}{c}
v^{\prime \prime}=0 \\
v(0)=v(\pi)
\end{array}\right\}
$$

Then it is known (see [21: Chapter 7]) that the boundary value problem $(P)_{q}$ is equivalent to the problem of finding $u \in C(0, \pi)$ such that

$$
u(t)=\int_{0}^{\pi} G(t, s)(g(s, u(s))+h(s) q(s)) d s .
$$

So, if we define the operator

$$
A_{q}(u)=\int_{0}^{\pi} G(t, s)(g(s, u(s))+h(s) q(s)) d s
$$

we are able to write the previous integral equation as

$$
u(t)=A_{q}(u)(t)
$$

In order to apply our main theorem we need to show that $\operatorname{deg}\left(I-A_{q}, B_{\rho}(0), 0\right) \neq 0$ for some (and hence for all) $q \in Q$, where the ball $B_{\rho}(0)$ plays the role of the set $\Omega$. To that aim let us consider the homotopy $H_{q}(\lambda, u)=u-\lambda A_{q}(u)(\lambda \in[0,1])$. This homotopy is an admissible one. To prove this statement it will be enough to show that $u \neq A_{q}(u)$ for all $u$ such that $\|u\|=\rho$ and $\lambda \in(0,1)$. That means that the integral equation

$$
u(t)=\lambda \int_{0}^{\pi} G(t, s)(g(s, u(s))+h(s) q(s)) d s
$$

has no solutions belonging to the boundary of the ball $B_{\rho}(0)$. The conditions we put on the function $g$ allow us to to say that $\|g(\cdot, u)\| \leq A\|u\|$ for some $A>0$. Afterward, since $\left\|h_{0}\right\|+|r|\|\phi\|=\left\|h_{0}\right\|+|r|$, for all $q \in Q$ we can write

$$
\|g(\cdot, u)+h q\| \leq A\|u\|+\left(\left\|h_{0}\right\|+|r|\right) \bar{M}=A\|u\|+B
$$

where we put $B=\left(\left\|h_{0}\right\|+|r|\right) \bar{M}$. Then, by a well-known result (see [16: p. 30]) we are allowed to say that there exists $M_{0}>0$ such that $\|u\| \leq M_{0}$ for any solution $u$ of the $\lambda$-integral equation. So any real number $\rho>M_{0}$ will be a good choice for the radius of $B_{\rho}(0)$. Finally, the above Theorem 2 (due to [14]) and the suitable definition of the set $Q$ allow to claim the existence of a solution of the problem (P). 


\section{References}

[1] Adimurthi and P. N. Srikanth: On exact number of solutions at infinity for AmbrosettiProdi class of problems. Bull. Un. Mat. Ital. 6 (1984), $15-24$.

[2] Ambrosetti, A. and G. Prodi: Analisi non lineare. Pisa: Quaderni della Scuola Normale Superiore 1973.

[3] Anichini, G.: Nonlinear problems for systems of differential equations. Nonlin. Anal. Meth. \& Appl. 1 (1976/77), 691 - 699.

[4] Anichini, G., Conti, G. and P. Zecca: Using solution sets for solving boundary value problems for ordinary differential equations. Nonlin. Anal. Meth. \& Appl. 5 (1991), 465 -472 .

[5] Anichini, G. and G. Conti: Existence of solutions of a boundary value problem through the solution map of a linearized type problem. Rend. Sem. Mat. Pol. Torino 48 (1990), $149-159$.

[6] Borsuk, K.: Theory of Retracts. Warszawa: Polish Sci. Publ. (PWN) 1967.

[7] Cecchi, M., Furi, M. and M. Marini: About asymptotic problems for ordinary differential equations. Boll. Un. Mat. Ital. (7) 2-B (1988), 333 - 343.

[8] Conti, G. and J. Pejsachowicz: Fixed point theorems for multivalued maps. Annali Matem. Pura Appl. (4) 126 (1980), 319 - 341.

[9] Conti, R.: Recent trends in the theory of boundary value problems for ordinary differential equations. Boll. Un. Mat. Ital. 22 (1967), 135 - 178.

[10] Costa, D. G., de Figueiredo, D. G. and P. N. Srikanth: The exact number of solutions for a class of ordinary differential equations through Morse index computation. J. Diff. Equ. 96 (1992), 185 - 199.

(11) Darbo, D.: Grado topologico e teoremi di esistenza per punti uniti per trasformazioni plurivalenti di bicelle. Rend. Sem. Mat. Univ. Padova 19 (1950), 371 - 395.

[12] Darbo, G.: Teoria dell'omologia in una categoria di mappe plurivalenti ponderate. Rend. Sem. Mat. Univ. Padova 28 (1958), 188 - 224.

[13] Deimling, K.: Nonlinear Functional Analysis. New York: Springer-Verlag 1985.

[14] Gallouët, T. and O. Kavian: Resultats d'existence et de non existence pour certaines problèmes demi-linéaires à l'infini. Ann. Fac. Sci. Toulouse 3 (1981), $201-246$.

[15] Granas, A., and Z. E. A. Guennoun: Quelques resultats dans la thèorie de BernsteinCarathèodory de l'equation $y^{\prime \prime}=f\left(t, y, y^{\prime}\right)$. Comptes Rend. Acad. Sci. 306 (1988), 703 706.

[16] Granas, A., Guenther, R. and J. Lee: Nonlinear Boundary Value Problems for Ordinary Differential Equations. Warszawa: Dissertationes Matematicae 244 (1985).

[17] Granas, A., Guenther, R. and J. Lee: Some general existence principles on the Carathèdory theory of nonlinear differential systems. J. Math. Pures Appl. 70 (1991), 153 196.

[18] Granas, A., Guenther, R. and J. Lee: Existence principles for classical and Carathéodory solutions of nonlinear systems and applications. In: Differential Equations and Applications, Vol I + II (ed.: A. R. Aftabizadeh). Columbus: Ohio Univ. Press 1988, pp. 353 364.

[19] Krasnoselkii, M. A.: Topological Methods in the Theory of Nonlinear Integral Equations. Berlin: Springer-Verlag 1964. 
[20] Pejsachowicz, J.: A Lefschetz type theorem for multivalued weighted mappings. Boll. Un. Mat. Ital. 14-A (1977), 391 - 397.

[21] Roach, G. F.: Green's Functions: Introductory Theory with Applications. London: Van Nostrand Reinhold 1970.

[22] Zeidler, E.: Nonlinear Functional Analysis and its Applications. Volume I: Fixed Point Theorems. New York: Springer-Verlag 1986.

Received 04.09.1996; in revised form 15.04.1997 